\title{
Co-treatment with Celecoxib or NS398 Strongly Sensitizes Resistant Cancer Cells to Antimitotic Drugs Independent of P-gp Inhibition
}

\author{
JONG SEUNG LIM, YUJIN PARK, BYUNG MU LEE, HYUNG SIK KIM and SUNGPIL YOON
}

School of Pharmacy, Sungkyunkwan University, Suwon, Republic of Korea

\begin{abstract}
Background/Aim: Inhibition of cyclooxygenase$2(C O X-2)$ has been investigated in clinical trials. Currently, NS398 and celecoxib are the most commonly used COX-2 inhibitors. The purpose of this study was to identify conditions that would increase the sensitivity of resistant cancer cells to antimitotic drugs. Materials and Methods: We tested whether COX-2 inhibitors can sensitize drug-resistant KBV20C cancer cells. We also compared the efficacy of NS398 with that of celecoxib. Results: Both NS398 and celecoxib could sensitize KB and KBV20C cells to a similar extent, suggesting that COX-2 inhibitors could be used for sensitive, as well as resistant, cancer cells. We demonstrated that the NS398 and celecoxib sensitization mechanism is independent of the inhibition of p-glycoprotein (P-gp), suggesting that resistant KBV2OC cells are sensitized through targeting of signaling pathways by both drugs. Furthermore, through using microscopic observation, assessment of cleaved poly $A D P$ ribose polymerase $(C$ $P A R P)$ and annexin $V$ staining we determined that both COX-2 inhibitors strongly sensitized resistant KBV20C cells to vinblastine (VIB) or paclitaxel (PAC) treatment. These results suggest that antimitotic drug-resistant cancer cells can be strongly sensitized by co-treatment with COX-2 inhibitors, without $P$-gp inhibitory activity. Conclusion: These findings provide important information regarding the sensitization of drug-resistant cells and indicate that COX-2 inhibitors may be used for potentially resistant cancer patients, without the toxic effects of P-gp inhibition.
\end{abstract}

This article is freely accessible online.

Correspondence to: Hyung Sik Kim, Ph.D. and Sungpil Yoon, Ph.D., School of Pharmacy, Sungkyunkwan University, 2066 Seobu-ro, Jangan-gu, Suwon, Gyeonggi-do, 16419, Republic of Korea. Tel: +82 312907789, Fax: +82 312928800, e-mail: hkims@skku.edu,syoon88@gmail.com

Key Words: COX-2, celecoxib, NS-398, P-gp, drug-resistant cancer.
Antimitotic drugs are widely used to treat numerous types of cancers $(1,2)$. These compounds inhibit mitosis by targeting microtubules and preventing their polymerization or depolymerization (1-4). However, patients develop resistance to these drugs (5). Thus, to improve the efficacy of treatment, research has been focused on increasing antimitotic drug-associated apoptosis.

Cyclooxygenase-2 (COX-2) is an enzyme whose expression increases in response to inflammation and mitotic stimuli (6-8). COX-2 expression is also positively correlated with cancer cell proliferation and growth (9). Increased COX-2 expression is also observed in P-glycoprotein (P-gp)mediated drug resistance $(10,11)$. There are reports that the COX-2 inhibitors NS398 and celecoxib can suppress cancer by COX-2-dependent and COX-2-independent mechanisms $(12,13)$. In addition, co-treatment with COX-2 inhibitors showed increased sensitization to anti-cancer drugs in various models (14-17). Adverse effects, such as cardiovascular events, have been reported in clinical trials involving celecoxib-treated cancer patients $(18,19)$. Better understanding of the mechanism governing the sensitization effect of COX-2 inhibitors in cancer patients could facilitate their safe therapeutic use.

In the present study, we compared the sensitization efficacy of two well-known COX-2 inhibitors, NS398 and celecoxib, in antimitotic drug-resistant KBV20C cancer cells. We also tested whether co-treatment with COX-2 inhibitors increases sensitization in antimitotic drug-treated KBV20C resistant cancer cells. Since we have already demonstrated their strong inhibitory effects in drug-resistant cancer, the current study supports the use of $\mathrm{COX}-2$ inhibitors in combinatorial treatment of antimitotic drug-resistant patients.

\section{Materials and Methods}

Reagents. Paclitaxcel (PAC) and verapamil (VER) were purchased from Sigma-Aldrich (St.Louis, MO, USA). Vinblastine (VIB) and vincristine (VIC) were purchased from Enzo Life Sciences (Farmingdale, NY, USA). Celecoxib and rhodamine123 (rhodamine) was purchased from Santa Cruz Biotechnology (Dallas, TX, USA). 
NS398 was purchased from Cayman Chemical (Ann Arbor, MI, USA). Calcein-AM was obtained from Invitrogen (Carlsbad, CA, USA).

Antibodies. Antibodies against pGSK3 $\beta$, pHistone H3, Cdc2, p21, PCNA and cleaved poly ADP ribose polymerase (C-PARP) were from Cell Signaling Technology (Danvers, MA, USA). Antibodies against glyceraldehyde 3-phosphate dehydrogenase (GAPDH), p27 and survivin were from Santa Cruz Biotechnology. Antibody against $\mathrm{pH} 2 \mathrm{AX}$ was from Abcam (Cambridge, UK).

Cell culturing. Human oral squamous carcinoma cell lines, KB and its multidrug-resistant subline, KBV20C, were obtained from Dr. Yong Kee Kim (College of Pharmacy, Sookmyung Women's University, Seoul, Republic of Korea) and have been previously described (2022). All cell lines were cultured in RPMI 1640 containing $10 \%$ fetal bovine serum, $100 \mathrm{U} / \mathrm{ml}$ penicillin and $100 \mu \mathrm{g} / \mathrm{ml}$ streptomycin (WelGENE, Daegu, South Korea).

Microscopic observation. Cells grown in 6-well plates were treated with the indicated drugs for the indicated times. The medium was removed and PBS was added in each dish. Cells were examined immediately in two independent experiments using an Axio observer.Z1 fluorescence inverted microscope (Carl Zeiss, Oberkochen, Germany) with a $5 \times$ or $10 \times$ objective lens (Carl Zeiss EC Plan-Neofluar).

Fluorescence-activated cell sorting (FACS) analysis. FACS analysis was performed as previously described (23-25). Cells were grown in $60-\mathrm{mm}$ diameter dishes and treated with the indicated drugs for the prescribed times. The cells were then dislodged by trypsin and pelleted by centrifugation. The pelleted cells were washed thoroughly with PBS, suspended in $75 \%$ ethanol for at least $1 \mathrm{~h}$ at $4^{\circ} \mathrm{C}$, washed with PBS and re-suspended in a cold propidium iodide (PI) staining solution $(100 \mu \mathrm{g} / \mathrm{ml}$ RNase A and $50 \mu \mathrm{g} / \mathrm{ml}$ PI in PBS $)$ for $30 \mathrm{~min}$ at $37^{\circ} \mathrm{C}$. The stained cells were analyzed in two independent experiments for relative DNA content using a FACSCalibur flow cytometry system (BD Bioscience, Franklin Lakes, NJ, USA).

Annexin $V$ analysis. Annexin $\mathrm{V}$ analysis was conducted using the annexin V-fluorescein isothiocyanate (FITC) staining kit (BD Bioscience) as previously described (23-25). Cells were grown in $60-\mathrm{mm}$ diameter dishes and treated with the indicated drugs for the prescribed times. The cells were then dislodged by trypsin and pelleted by centrifugation. The pelleted cells were washed with PBS. Cells in $100 \mu \mathrm{l}$ of binding buffer received $5 \mu \mathrm{l}$ of Annexin VFITC and $5 \mu \mathrm{l}$ of PI and were, then, incubated for $15 \mathrm{~min}$ at room temperature. The stained cells were analyzed in two independent experiments using a FACSCalibur flow cytometry system (BD Bioscience).

Rhodamine and calcein-AM uptake tests. The tests used for determination of ability for inhibition of P-gp are based on a previously described method (23-25). Briefly, cells grown in 6-well plates were treated with indicated drugs and incubated for $24 \mathrm{~h}$ at $37^{\circ} \mathrm{C}$. Cells were then incubated with and $1 \mu \mathrm{g} / \mathrm{ml}$ rhodamine or 0.1 $\mu \mathrm{g} / \mathrm{ml}$ calcein- $\mathrm{AM}$ for $1 \mathrm{~h} 30 \mathrm{~min}$ at $37^{\circ} \mathrm{C}$. The medium were removed and the cells were washed with PBS. The stained cells were analyzed in two independent experiments using a FACSCalibur flow cytometry system (BD Bioscience).
Western blot analysis. Total cellular proteins were extracted using a previously described trichloroacetic acid (TCA) method (23-25). Briefly, cells grown in 60-mm dishes were washed three times with $5 \mathrm{ml}$ PBS. Next, $500 \mu \mathrm{l}$ of $20 \%$ TCA was added to each plate. The cells were then dislodged by scraping and transferred to Eppendorf tubes. Proteins were pelleted by centrifugation for $5 \mathrm{~min}$ at 3,000 $\mathrm{rpm}$ and re-suspended in $1 \mathrm{M}$ Tris- $\mathrm{HCl}(\mathrm{pH} 8.0)$ buffer. The total protein concentrations were estimated. The proteins were resolved by sodium dodecyl sulfate-polyacrylamide gel electrophoresis (SDS-PAGE) and subjected to Western blot analysis as previously described (23-25).

\section{Results}

COX-2 inhibitors are not substrates for P-gp in drugresistant $K B V 20 C$ cells. The importance of COX-2 inhibitors in cancer therapy has been documented $(6,7)$. However, the relative efficacy of different COX-2 inhibitors in cancer treatment has not been compared. We, thus, tested whether the COX-2 inhibitors NS398 and celecoxib could sensitize KBV20C cells, that have an antimitotic drug-resistant phenotype. NS398 and celecoxib have been widely studied for clinical applications in cancer therapy $(11,12,14)$. We also tested whether drug-resistant KBV20C cells have resistance to these drugs. Cellular growth was microscopically observed after $48 \mathrm{~h}$ of treatment with each drug in both sensitive parent KB and resistant KBV20C cells. As seen in Figure 1A-B, KBV20C cells were highly resistant to the anticancer drug VIC, while both $\mathrm{KB}$ and KBV20C cells exhibited a similar level of sensitization by NS398 or celecoxib. This result suggests that NS398 and celecoxib are not substrates of $\mathrm{P}$-gp in drug-resistant KBV20C cells. It also suggests that COX-2 inhibitors can be used as an anticancer drug in resistant cells. NS398 and celecoxib have different structures, suggesting that our findings may be evident with other COX-2 inhibitors.

We also assessed whether NS398 and celecoxib inhibit Pgp. Our results showed that NS398 and celecoxib did not inhibit P-gp activity, as seen with anticancer drugs VIB and PAC (Figure 1C). However, P-gp inhibition was observed when using a well-known P-gp inhibitor, verapamil (Figure 1C). Thus, we conclude that COX-2 inhibitors sensitize resistant KBV20C cells via cellular signaling pathways.

Co-treatment with COX-2 inhibitors strongly increases toxicity of antimitotic drugs in resistant KBV20C cells without P-gp inhibition. Although we did not observe P-gp inhibitory activity by COX-2 inhibitors (Figure 1C), we tested whether co-treatment with NS398 or celecoxib could increase sensitization of antimitotic drug-treated KBV20C cells. As seen in Figure 2A, co-treatment with NS398 or celecoxib reduced proliferation of both VIB- and PACtreated KBV20C cells. Considering that these antimitotic drugs have different modes of action, with VIB targeting the 
A

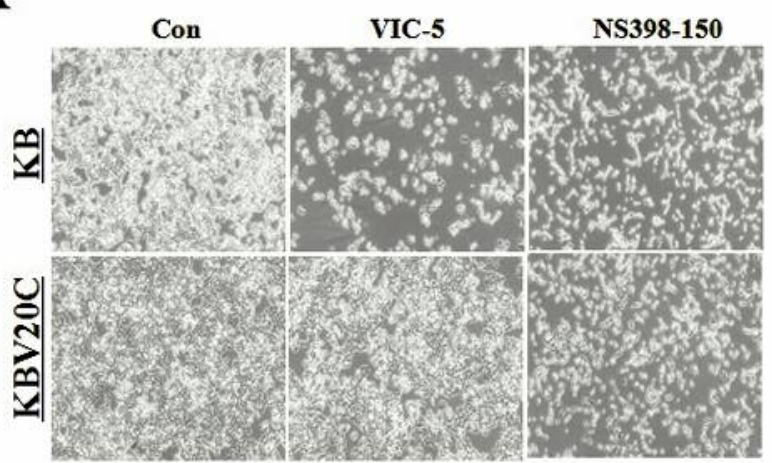

B

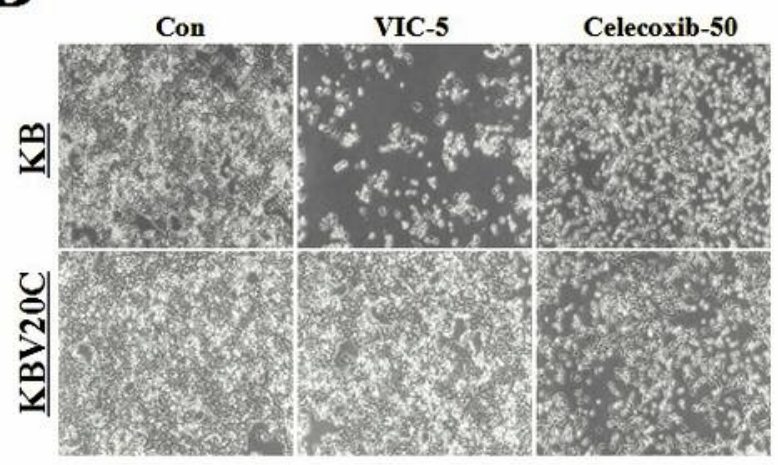

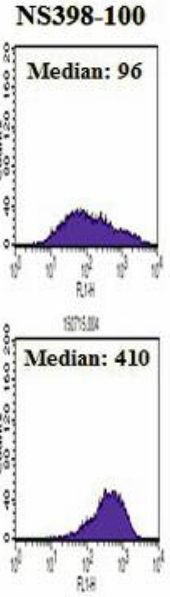

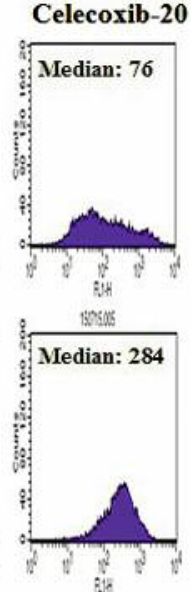

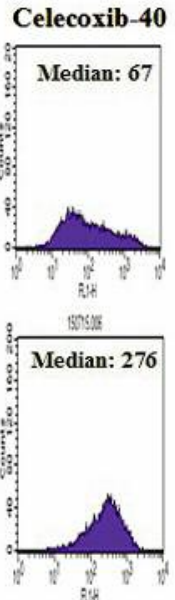

Figure 1. COX-2 inhibitors are not substrates for P-gp in drug-resistant KBV20C cells. $(A-B)$ KB and KBV20C cells were grown on 6-well plates and treated with $5 \mathrm{nM}$ vincristine (VIC-5), $150 \mu \mathrm{M}$ NS398 (NS398-150), $50 \mu \mathrm{M}$ celecoxib (Celecoxib-50) or 0.1\% DMSO (Con). After 2 days, all cells were observed using an inverted microscope with a 50 magnification. (C) KBV20C cells were grown on 60 mm-diameter dishes and treated with $10 \mu M$ verapamil (VER-10), $5 \mathrm{nM}$ vinblastine (VIB-5), $30 \mathrm{nM}$ paclitaxcel (PAC-30), $50 \mu \mathrm{M}$ NS398 (NS398-50), 100 $\mu$ M NS398 (NS398-100), $20 \mu M$ celecoxib (Celecoxib-20), $40 \mu M$ celecoxib (Celecoxib-40) or 0.1\% DMSO (Con). After 24 h, all cells were stained with rhodamine or calcein-AM, as described in the Materials and Methods section. The stained cells were subsequently examined by FACS analysis.

vinca domain and PAC targeting the taxane-binding site of microtubules, we hypothesized that these results could be observed with various kinds of antimitotic drugs.

In a more detailed analysis using fluorescence-activated cell sorting, all co-treatments (VIB-NS398, VIB-celecoxib, PAC-NS398 and PAC-celecoxib) showed increased G2 arrest when compared with that observed upon monotherapy with each drug (Figure 2B). The finding suggests that the reduced proliferation observed upon the use of COX-2 inhibitors resulted from increased $\mathrm{G} 2$ cell cycle arrests. Because we observed an increased pre-G1 region in fluorescence-activated cell sorting (Figure 2B), we also performed annexin $\mathrm{V}$ staining. As seen in Figure $2 \mathrm{C}$, early apoptotic events were increased by co-treatment. We confirmed our results with western blot analysis, wherein C-PARP production greatly increased due to VIB co-treatments (Figure 3A). We conclude that COX-2 inhibitors increased apoptosis in VIB- and PAC-treated KBV20C cells.

We also observed that the number of apoptotic events increased with an increase in treatment duration. As seen in Figure $3 \mathrm{~B}-\mathrm{C}$, the pre- $\mathrm{G}_{1}$ region and early apoptotic events were greatly increased when drug treatment was continued for 2 days. The finding suggests that co-treatment can maintain toxicity over a longer period, with greater numbers of cells being irreversibly damaged.

We assumed that co-treatment with NS398 or celecoxib could restore cytotoxicity in antimitotic drug-treated KBV20C cells. Since COX-2 inhibitors are not involved in inhibition of $\mathrm{P}$-gp activity, it is evident, from Figure $1 \mathrm{C}$, that 

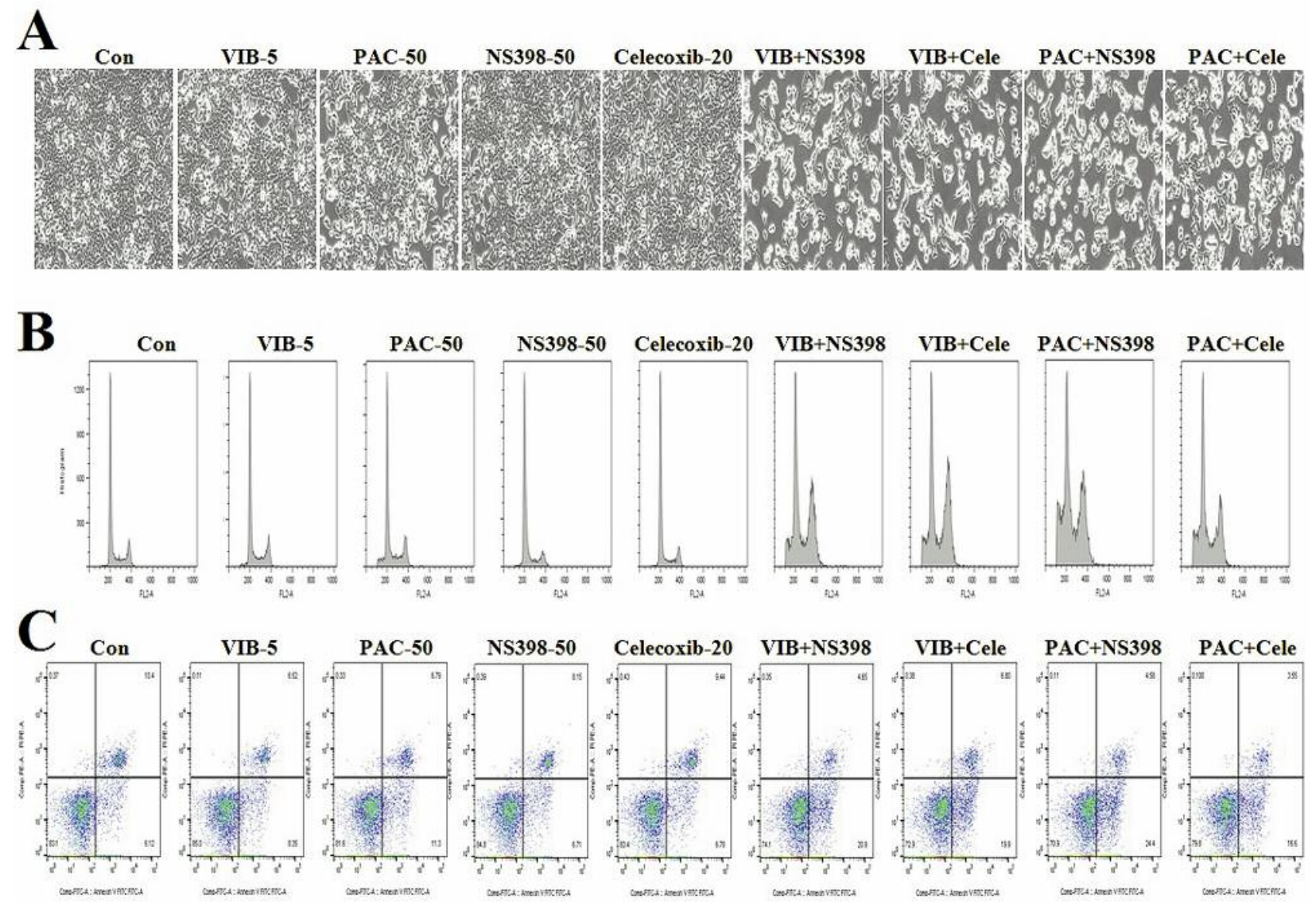

Figure 2. Co-treatment with COX-2 inhibitors strongly recovers the toxicity of antimitotic drugs in resistant KBV20C cells. (A-C) KBV20C cells were grown on 6-well plates or $60 \mathrm{~mm}$-diameter dishes and treated with $5 \mathrm{nM}$ vinblastine (VIB-5), $50 \mathrm{nM}$ paclitaxcel (PAC-50), $50 \mu \mathrm{M}$ NS398 (NS398-50), $20 \mu M$ celecoxib (Celecoxib-20), $5 \mathrm{nM}$ vinblastine with $50 \mu \mathrm{M}$ NS398 (VIB+NS398), $5 \mathrm{nM}$ vinblastine with $20 \mu \mathrm{M}$ celecoxib (VIB+Cele), $50 \mathrm{nM}$ paclitaxcel with $50 \mu \mathrm{M}$ NS398 (PAC+NS398), $50 \mathrm{nM}$ paclitaxcel with $20 \mu \mathrm{M}$ celecoxib (PAC + Cele) or $0.1 \%$ DMSO (Con). After 2 days, all cells were observed using an inverted microscope with a 100 magnification (A). After 24 h, FACS (B) and Annexin V (C) analyses were performed as described in the Materials and Methods section.

VIB-NS398 and VIB-celecoxib sensitization in KBV20C cells involves intracellular pathways to recover NS398 and celecoxib to increase sensitization. We have also determined the most effective combination of COX-2 inhibitors and antimitotic drugs. Using various techniques, such as microscopic observations, fluorescence-activated cell sorting and C-PARP production analysis (Figure 2A-3C), we could identify effective co-treatments. However, after analyzing the obtained data, we conclude that there was no significant difference in efficacy between any of the treatment combinations. Considering that both NS398 and celecoxib have different molecular structures for targeting COX-2 inhibition, we conclude that co-treatment with other COX-2 inhibitors to sensitize drug-resistant cancer cells may be similarly effective.
Celecoxib has more sensitization activity than NS398 in cotreated sensitive $K B$ cells. To determine the molecular mechanisms underlying the NS398- or celecoxib-associated increase in VIB-induced toxicity in KBV20C cells, we tested whether co-treatment with VIB and COX-2 inhibitors influences the activation or expression of signaling proteins. When we assessed the levels of important proliferationrelated proteins or phosphorylation levels $(26,27)$, we did not identify major signal changes (Figure 4A), suggesting that a more detailed analysis may be needed for further identification of important signaling proteins in co-treatments.

Sensitive KB and resistant KBV20C cells exhibit similar sensitization by monotherapy with COX-2 inhibitors (Figure 1A-B). Additionally, we tested whether KB cells also showed increased sensitization upon combination 


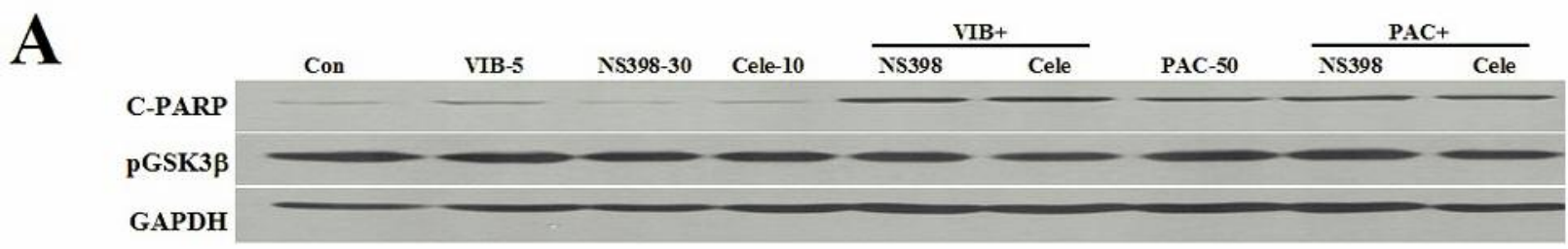

B
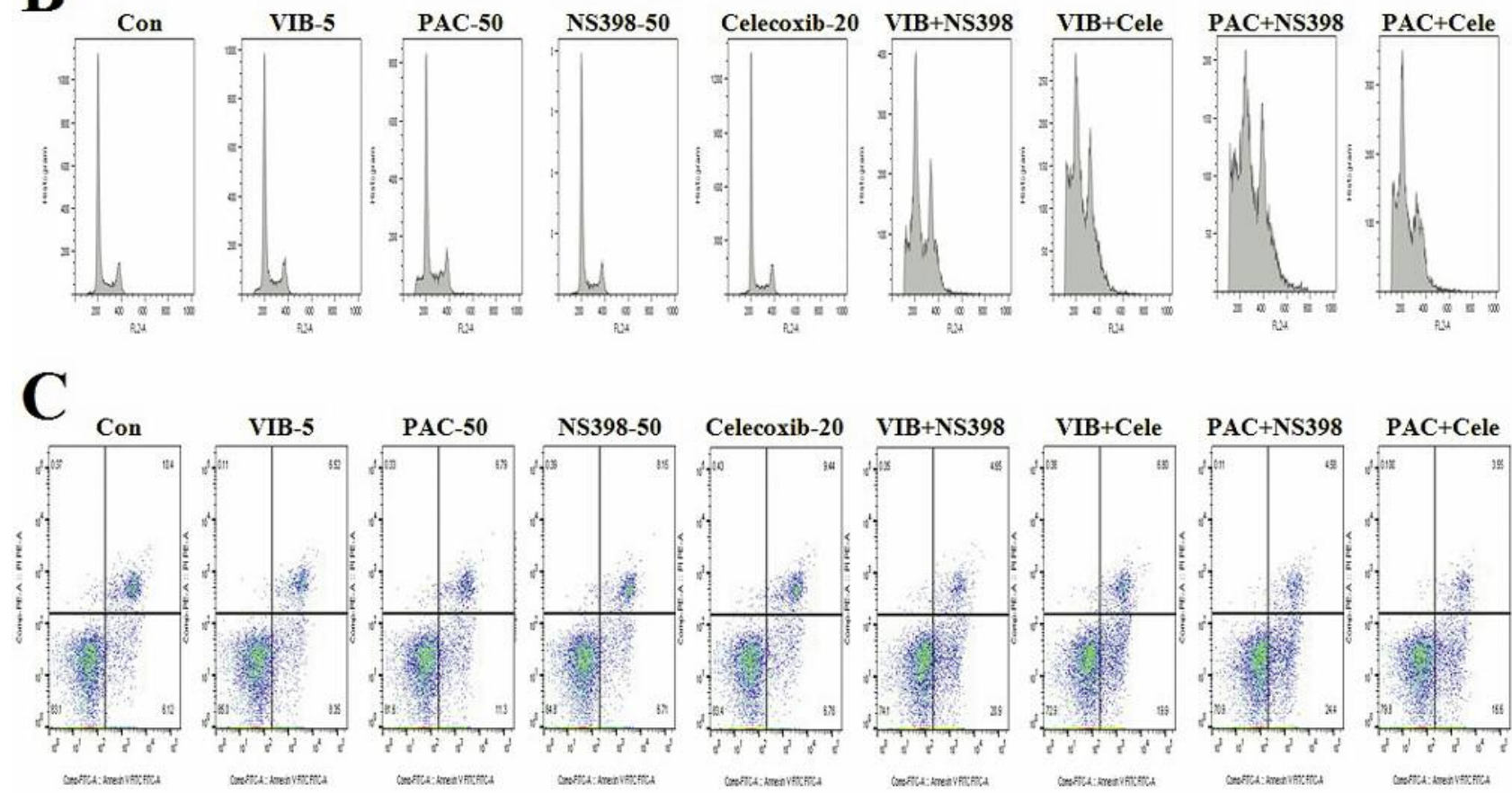

Figure 3. Co-treatment with COX-2 inhibitors greatly increases apoptosis of antimitotic drug-treated resistant KBV20C cells. (A) KBV20C cells were grown on $60 \mathrm{~mm}$-diameter dishes and treated with $5 \mathrm{nM}$ vinblastine (VIB-5), $50 \mathrm{nM}$ paclitaxcel (PAC-50), $30 \mu \mathrm{M}$ NS398 (NS398-30), $10 \mu \mathrm{\mu M}$ celecoxib (Celecoxib-10), $5 \mathrm{nM}$ vinblastine with $30 \mu \mathrm{M}$ NS398 (VIB+NS398), $5 \mathrm{nM}$ vinblastine with $10 \mu \mathrm{M}$ celecoxib (VIB+Cele), $50 \mathrm{nM}$ paclitaxcel with $30 \mu M$ NS398 (PAC+NS398), $50 \mathrm{nM}$ paclitaxcel with $10 \mu \mathrm{M}$ celecoxib (PAC+Cele) or 0.1\% DMSO (Con). After 24 h, Western blot analysis was performed using antibodies against C-PARP and GAPDH. (B-C) KBV20C cells were grown on $60 \mathrm{~mm}$-diameter dishes and treated with $5 \mathrm{nM}$ vinblastine (VIB-5), $50 \mathrm{nM}$ paclitaxcel (PAC-50), $50 \mu \mathrm{M}$ NS398 (NS398-50), $20 \mu \mathrm{M}$ celecoxib (Celecoxib-20), $5 \mathrm{nM}$ vinblastine with $50 \mu \mathrm{M}$ NS398 (VIB+NS398), 5 nM vinblastine with $20 \mu \mathrm{M}$ celecoxib (VIB+Cele), $50 \mathrm{nM}$ paclitaxcel with $50 \mu \mathrm{M}$ NS398 (PAC+NS398), $50 \mathrm{nM}$ paclitaxcel with $20 \mu \mathrm{M}$ celecoxib (PAC+Cele) or $0.1 \%$ DMSO (Con). After 2 days, FACS (B) and Annexin V (C) analyses were performed as described in the Materials and Methods section.

treatment with VIB-NS398 or VIB-celecoxib. As seen in Figure 4B, VIB-celecoxib exhibited more sensitization than VIB-NS398, suggesting that celecoxib is more effective than NS398 in treating sensitive KB cells when used in combination with antimitotic drugs. We also confirmed that this increased sensitization occurs upon co-treatment with VIC (Figure 4C). This finding implies that cell line specificity for different COX-2 inhibitors is possible but resistant cancer cells have similar sensitization specificity for both NS398 and celecoxib, thus suggesting that COX2 inhibitors have common sensitization mechanisms for resistant cancer cells.

\section{Discussion}

The importance of COX-2 inhibitors in cancer treatment has been previously demonstrated $(6,7,18,19)$. There are two well-known inhibitors, NS398 and celecoxib, that have been investigated in various cancer models, including drugresistant cancer cells, as potential cancer treatments $(11,12$, 14). However, studies comparing different COX-2 inhibitors in drug-resistant cancer models have not been conducted. It is important to test which type of drug can sensitize specific cell types for their optimal application in the clinical setting. In this study, we investigated the sensitization efficacy of 

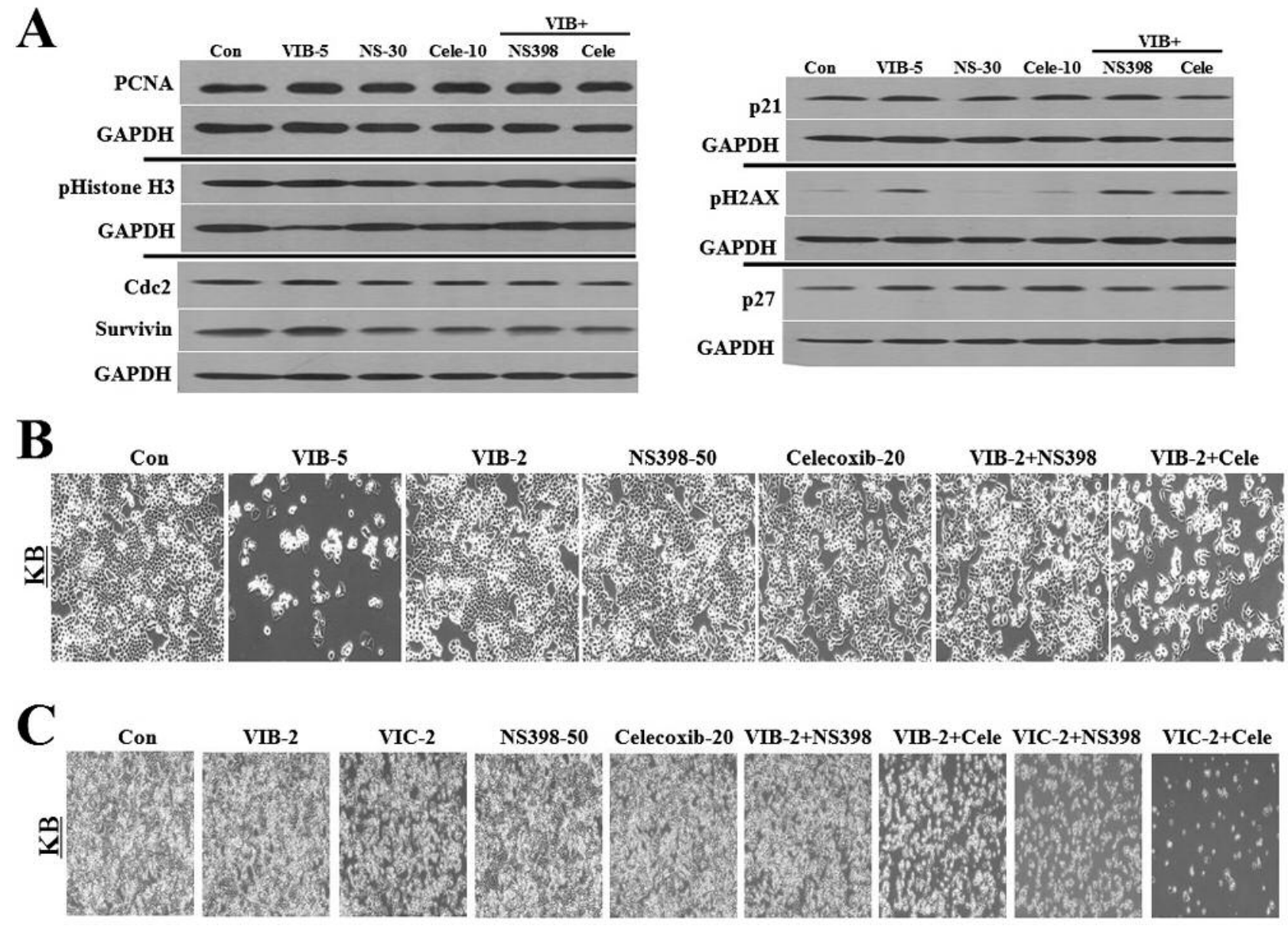

Figure 4. Celecoxib has greater sensitization activity than that of NS398 in co-treated sensitive KB cells. (A) KBV20C cells were grown on 60 mmdiameter dishes and treated with $5 \mathrm{nM}$ vinblastine (VIB-5), $30 \mu \mathrm{M}$ NS398 (NS-30), $10 \mu \mathrm{M}$ celecoxib (Cele-10), $5 \mathrm{nM}$ vinblastine with $30 \mu \mathrm{M}$ NS398 (VIB+NS398), $5 \mathrm{nM}$ vinblastine with $10 \mu \mathrm{M}$ celecoxib (VIB+Cele) or 0.1\% DMSO (Con). After $24 \mathrm{~h}$, western blot analysis was performed using antibodies against PCNA, pHistone H3, Cdc2, survivin, p21, pH2AX, p27 and GAPDH. $(B-C) \mathrm{KB}$ cells were grown on 6-well plates and treated with $5 \mathrm{nM}$ vinblastine (VIB-5), $2 \mathrm{nM}$ vinblastine (VIB-2), $2 \mathrm{nM}$ vincristine (VIC-2), $50 \mu M$ NS398 (NS398-50), 20 $\mu$ M celecoxib (Celecoxib-20), $2 \mathrm{nM}$ vinblastine with $50 \mu \mathrm{M}$ NS398 (VIB-2+NS398), 2 nM vinblastine with $20 \mu \mathrm{M}$ celecoxib (VIB-2+Cele), 2 nM vincristine with $50 \mu M$ NS398 (VIC-2+NS398), $2 \mathrm{nM}$ vincristine with $20 \mu \mathrm{M}$ celecoxib (VIC-2+Cele) or $0.1 \%$ DMSO (Con). After 1 day, all cells were observed using an inverted microscope with a $x 100$ magnification (B). After 2 days, all cells were observed using an inverted microscope with a x50 magnification (C).

NS398 and celecoxib in antimitotic drug-resistant KBV20C cancer cells. These drugs are already used in clinical settings. Thus, once their mechanism of action in cancer cells is known, these drugs could be readily available for use without further toxicity studies.

We found that NS398 and celecoxib have similar sensitization effects in drug-resistant cancer cells. KBV20C cells are highly resistant to anticancer drugs, such as VIC. However, both COX-2 inhibitors sensitized drug-resistant KBV20C cells and parent sensitive KB cells to a similar degree. These results suggest that COX-2 inhibitors are not substrates for P-gp-mediated efflux pumping. Our data also confirmed that neither of the two drugs affected P-gp activity in KBV20C cells.

Next, we tested whether co-treatment with NS398 or celecoxib could increase sensitization in combination with VIB or PAC in resistant KBV20C cells. We found that cotreatment with NS398 or celecoxib sensitized both VIB- and PAC-treated KBV20C cells. We confirmed that the KBV20C-specific sensitization was independent of P-gp inhibition when using two different P-gp substrates, calceinAM and rhodamine. The results suggest that NS398 and celecoxib target cellular signaling pathways specific to antimitotic drug-resistant cancer cells. The sensitization 
mechanism involves an increase in $\mathrm{G}_{2}$-phase cell-cycle arrest in KBV20C cells. It is assumed that patients who are resistant to antimitotic drugs could be treated with NS398 or celecoxib to effectively increase $\mathrm{G}_{2}$-phase cell-cycle arrest. COX-2 inhibitors can be considered and categorized as drugs that enhance the activity of antimitotic drugs in resistant cancer cells without P-gp inhibition. The increase in $\mathrm{G}_{2^{-}}$ phase cell-cycle arrest by $\mathrm{COX} 2$ inhibitors finally resulted in increased apoptosis, which was confirmed by an increase in pre- $\mathrm{G}_{1}$ phase cell-cycle arrest, $\mathrm{C}$-PARP production and annexin $\mathrm{V}$ staining. Additionally, the COX-2 inhibitor sensitization effect on KBV20C cells is time- and dosedependent showing that co-treatment can maintain toxicity over a prolonged period, with greater numbers of cells being irreversibly damaged. We also demonstrated that two different antimitotic drugs, VIB and PAC, have similar efficacy when combined with either NS398 or celecoxib for sensitization. Considering that the antimitotic drug VIB targets the vinca domain and PAC targets the taxane-binding site of microtubules, we assumed that the results could be replicated for various kinds of antimitotic drugs.

Since both NS398 and celecoxib have different structures and similar functional activity to target COX-2 inhibition, we conclude that our findings are conserved in COX-2 inhibitors and can be applied to other COX-2 inhibitors. In the future, it will be important to determine whether other resistant cancer cell lines, including different organ-derived cancer cells, also exhibit the same sensitivity to COX-2 inhibitors. The molecular targets for the strong sensitization effects of co-treatment with COX-2 inhibitors and antimitotic drugs could be one of the important issues to address in further studies. Future studies using an in vivo mouse model are warranted for assessing the sensitization effect and toxicity of COX-2 inhibitors.

Our work demonstrated that two COX-2 inhibitors could sensitize both resistant and sensitive cancer cells without Pgp inhibition. We also provided evidence that both COX-2 inhibitors have applications in the treatment of drug-resistant cancer patients. We found that both NS398 and celecoxib, as strong sensitizing drugs in antimitotic drug-treated resistant cancer cells, increased apoptosis via $\mathrm{G}_{2}$-phase cell-cycle arrest. Since COX-2 inhibitors have been shown to have a sensitization effect on cancer independent of COX-2 inhibition $(12,13)$, we propose that our results can be applied to other drugs targeting resistant cancer cells, irrespective of whether they are COX-2 inhibitors or not. Our study may help improve COX-2 inhibitor-based chemotherapeutic treatments for cancer patients who develop resistance to anticancer drugs.

\section{Conflicts of Interest}

The Authors declare that no conflicts of interest exist.

\section{Acknowledgements}

The Authors would like to thank Ae-Ran Choi and Ju-Hwa Kim for help in technical supports and preparation of the manuscript. This research was supported by the National Research Foundation of Korea (NRF) funded by the Korean goverment (NRF2016R1A2B2011071).

\section{References}

1 Jordan MA and Wilson L: Microtubules as a target for anticancer drugs. Nat Rev Cancer 4: 253-265, 2004.

2 Yang $\mathrm{H}$ and Dou QP: Targeting apoptosis pathway with natural terpenoids: Implications for treatment of breast and prostate cancer. Curr Drug Targets 11: 733-744, 2010.

3 Matsuo K, Bond VK, Im DD and Rosenshein NB: Prediction of chemotherapy response with platinum and taxane in the advanced stage of ovarian and uterine carcinosarcoma: A clinical implication of in vitro drug resistance assay. Am J Clin Oncol 33: 358-363, 2010.

4 McGrogan BT, Gilmartin B, Carney DN and McCann A: Taxanes, microtubules and chemoresistant breast cancer. Biochim Biophys Acta 1785: 96-132, 2008.

5 Araque Arroyo P, Ubago Perez R, Cancela Diez B, Fernandez Feijoo MA, Hernandez Magdalena J and Calleja Hernandez MA: Controversies in the management of adjuvant breast cancer with taxanes: review of the current literature. Cancer Treat Rev 37: 105-110, 2011.

6 Cerella C, Sobolewski C, Dicato M and Diederich M: Targeting COX-2 expression by natural compounds: a promising alternative strategy to synthetic COX-2 inhibitors for cancer chemoprevention and therapy. Biochem Pharmacol 80: 18011815,2010 .

7 Ghosh N, Chaki R, Mandal V and Mandal SC: COX-2 as a target for cancer chemotherapy. Pharmacol Rep 62: 233-244, 2010.

8 Suganuma M, Saha A and Fujiki H: New cancer treatment strategy using combination of green tea catechins and anticancer drugs. Cancer Sci 102: 317-323, 2011.

9 Generali D, Buffa FM, Deb S, Cummings M, Reid LE, Taylor M, Andreis D, Allevi G, Ferrero G, Byme D, Martinotti M, Bottini A, Harris AL, Lakhani SR and Fox SB: COX-2 expression is predictive for early relapse and aromatase inhibitor resistance in patients with ductal carcinoma in situ of the breast, and is a target for treatment. Br J Cancer 111: 46-54, 2014.

10 Rahman M, Selvarajan K, Hasan MR, Chan AP, Jin C, Kim J, Chan SK, Le ND, Kim YB and Tai IT: Inhibition of COX-2 in colon cancer modulates tumor growth and MDR-1 expression to enhance tumor regression in therapy-refractory cancers in vivo. Neoplasia 14: 624-633, 2012.

11 Sui H, Zhou S, Wang Y, Liu X, Zhou L, Yin P, Fan Z and Li Q: COX-2 contributes to P-glycoprotein-mediated multidrug resistance via phosphorylation of c-Jun at Ser63/73 in colorectal cancer. Carcinogenesis 32: 667-675, 2011.

12 Amir M and Agarwal HK: Role of COX-2 selective inhibitors for prevention and treatment of cancer. Pharmazie 60: 563-570, 2005.

13 Lee NO, Park JW, Lee JA, Shim JH, Kong SY, Kim KT and Lee YS: Dual action of a selective cyclooxygenase-2 inhibitor on vascular endothelial growth factor expression in human hepatocellular carcinoma cells: Novel involvement of discoidin domain receptor 2. J Cancer Res Clin Oncol 138: 73-84, 2012. 
14 Cao Y, Qu J, Li C, Yang D, Hou K, Zheng H, Liu Y and Qu X: Celecoxib sensitizes gastric cancer to rapamycin via inhibition of the Cbl-b-regulated PI3K/Akt pathway. Tumour Biol 36: 5607-5615, 2015 .

15 Wang X, Zhang L, O'Neill A, Bahamon B, Alsop DC, Mier JW, Goldberg SN, Signoretti S, Atkins MB, Wood CG and Bhatt RS: Cox-2 inhibition enhances the activity of sunitinib in human renal cell carcinoma xenografts. Br J Cancer 108: 319-326, 2013.

$16 \mathrm{Xu}$ HB, Shen FM and Lv QZ: Celecoxib enhanced the cytotoxic effect of cisplatin in chemo-resistant gastric cancer xenograft mouse models through a cyclooxygenase-2-dependent manner. Eur J Pharmacol 776: 1-8, 2016.

17 Zhang H, Li Z and Wang K: Combining sorafenib with celecoxib synergistically inhibits tumor growth of non-small cell lung cancer cells in vitro and in vivo. Oncol Rep 31: 1954-1960, 2014.

18 Chen J, Shen P, Zhang XC, Zhao MD, Zhang XG and Yang L: Efficacy and safety profile of celecoxib for treating advanced cancers: A meta-analysis of 11 randomized clinical trials. Clin Ther 36: 1253-1263, 2014.

19 Legge F, Paglia A, D'Asta M, Fuoco G, Scambia G and Ferrandina G: Phase II study of the combination carboplatin plus celecoxib in heavily pre-treated recurrent ovarian cancer patients. BMC Cancer 11: 214, 2011

20 Choi AR, Kim JH, Woo YH, Kim HS and Yoon S: Anti-malarial drugs primaquine and chloroquine have different sensitization effects with anti-mitotic drugs in resistant cancer cells. Anticancer Res 36: 1641-1648, 2016.

21 Hwang JW, Cho H, Lee JY, Jeon Y, Kim SN, Lee SJ, Bae GU, Yoon S, Jeon R and Kim YK: The synthetic ajoene analog SPA3015 induces apoptotic cell death through crosstalk between NF-kappaB and PPARgamma in multidrug-resistant cancer cells. Food Chem Toxicol 96: 35-42, 2016.
$22 \mathrm{Kim}$ NH, Kim SN, Oh JS, Lee S and Kim YK: Anti-mitotic potential of 7-diethylamino-3(2'-benzoxazolyl)-coumarin in 5fluorouracil-resistant human gastric cancer cell line snu620/5-fu. Biochem Biophy Res Commun 418: 616-621, 2012.

23 Choi AR, Jo MJ, Jung MJ, Kim HS and Yoon S: Selenate specifically sensitizes drug-resistant cancer cells by increasing apoptosis via $\mathrm{G} 2$ phase cell cycle arrest without P-GP inhibition. Eur J Pharmacol 764: 63-69, 2015.

24 Choi AR, Jung MJ, Kim JH and Yoon S: Co-treatment of salinomycin sensitizes AZD5363-treated cancer cells through increased apoptosis. Anticancer Res 35: 4741-4747, 2015.

25 Choi AR, Kim JH and Yoon S: Thioridazine specifically sensitizes drug-resistant cancer cells through highly increase in apoptosis and P-gp inhibition. Tumour Biol 35: 9831-9838, 2014.

26 Choi AR, Kim JH and Yoon S: Sensitization of cancer cells through reduction of total AKT and downregulation of salinomycin-induction of pAKT, pGSK3beta, pTSC2, and p4EBP1 by cotreatment with MK-2206. Biomed Res Int 2014: 295760, 2014.

27 Kim JH, Choi AR, Kim YK, Kim HS and Yoon S: Low amount of salinomycin greatly increases AKT activation, but reduces activated p70S6K levels. Int J Mol Sci 14: 17304-17318, 2013.

Received August 18, 2016

Revised September 1, 2016

Accepted September 2, 2016 